\title{
Lipid-mediated regulation of flowering time
}

An anionic phospholipid tells plants when to flower based on ambient temperature By Yvon Jaillais ${ }^{1}$ and François Parcy ${ }^{2}$

${ }^{1}$ Laboratoire Reproduction et Développement des Plantes, Univ Lyon, ENS de Lyon, CNRS, INRAE, Lyon, France. ${ }^{2}$ Laboratoire Physiologie Cellulaire et Végétale, Univ. Grenoble Alpes, CNRS, CEA, INRAE, IRIGDBSCI-LPCV, Grenoble, France.

Email: yvon.jaillais@ens-lyon.fr

FLOWERING LOCUS T (FT) is a mobile protein, known as florigen, that induces flowering in plants. In Arabidopsis thaliana, FT transcription is induced in the leaf vascular tissues (phloem) when days become longer (1). It then moves at long distance through the phloem to reach the shoot apical meristem, where it induces floral transitions by regulating nuclear transcription complexes $(1,2)$. FT has homologies with characterized lipid-binding proteins in animals and belongs to the phosphatidylethanolamine-binding protein (PEBP) family (2). Although conserved throughout flowering plants, the function of FT lipid-binding capacity has remained enigmatic. On page XXX of this issue, Susila et al. (3) show that FT binds to the anionic phospholipid phosphatidylglycerol, which sequesters FT in membrane compartments of phloem cells at low temperature, thereby delaying flowering in cold conditions. Thus, a temperature-dependent regulation of FT translocation by a membrane phospholipid tunes the plant response to ambient temperature.

Susila et al. found that FT also binds to the anionic phospholipid phosphatidylglycerol in vitro and in phloem cells in vivo. Anionic phospholipids modulate the physicochemical properties of membranes, notably their electrostatic field, and provide docking platforms for proteins (4). Each anionic phospholipid species localizes to precise compartments within plant cells, thereby regulating the membrane identity of these organelles (4). Phosphatidylglycerol mostly accumulates in chloroplast and mitochondria (5), but has also been found in various compartments of the endomembrane system (6). However, its precise localization is largely unknown, raising the question of where FT interacts with phosphatidylglycerol.

Susila et al.. found that, in $A$. thaliana protoplasts, FT associates with various membranes that contain phosphatidylglycerol: plasma membrane, Golgi apparatus, trans-Golgi network, endoplasmic reticulum, and the chloroplast envelop. Low temperature (i.e. long day at $16^{\circ} \mathrm{C}$ ) induced the interaction between FT and cellular membranes in a phosphatidylglycerol-dependent manner.

Differentiated phloem tissues consist of conducting cells, called the sieve elements, and specialized parenchymal cells, called the companion cells (see the figure). Sieve elements are enucleated cells responsible for transporting sugars, metabolites and some proteins throughout the plants. Companion cells are nucleated cells responsible for the metabolic functioning of sieve elements. They are closely associated with sieve elements via specialized cell-to-cell connections called plasmodesmata. FT is expressed in 
phloem companion cells and then moves to the sieve elements via the cytoplasmic canal of the plasmodesmata (1, 7). Low temperature promotes the phosphatidylglycerol-dependent retention of FT in cellular membranes of companion cells, which delays flowering by reducing FT movement toward the sieve elements and thus the shoot meristem. In phosphatidylglycerol biosynthetic mutants, this temperaturedependent retention is reduced, leading to an early flowering phenotype and temperature insensitivity. During long days at $23^{\circ} \mathrm{C}$, FT is not sequestered within companion cell membranes and moves into phloem sieve elements to reach the shoot apical meristem and accelerate flowering.

Once in the meristem, FT binds to another phospholipid, phosphatidylcholine, to induce flowering $(5,8)$. FT preferentially interacts with phosphatidylcholine species with low unsaturation degree (i.e. few numbers of carbon-carbon double bonds in their fatty acid chains), which are more abundant under long day conditions. However, it remains unclear how ambient temperature modulates FTphosphatidylglycerol interaction and thus its mobility. Lipidomic analyses by Susila et al. suggest that the overall amounts of phosphatidylglycerol do not vary with temperature. Yet, consistent with the intense temperature-dependent remodeling of lipids in plants $(6,9,10)$, the abundance of polyunsaturated phosphatidylglycerol species increase at $16^{\circ} \mathrm{C}$ compared with $23^{\circ} \mathrm{C}$. Since FT interaction with phosphatidylcholine is dependent on its unsaturation degree (8), it could also be the case for FTphosphatidylglycerol interaction. As such, the membrane network, through changes in lipid unsaturation, could catch or release PEBP proteins, thereby regulating plant architecture in response to environmental cues, notably temperature variations. However, such a model will require further investigation.

A major step toward understanding how different phospholipid molecular species could regulate FT will be to characterize this interaction at the atomic level. High-resolution crystal structures suggest possible lipid binding sites (11), but the next step will be to analyze FT structure in complex with its lipid ligand or even directly inserted into membranes. Dissecting this interaction should allow the identification of FT variants specifically defective in lipid binding with intact transcriptional activity, thus allowing the uncoupling of temperature sensing capacity from the flowering inducing functions of FT. It will be particularly interesting to analyze whether such FT variants may already exist in nature, for example in plant species with temperature-insensitive flowering transition.

The results of Susila et al. provide a likely explanation for why florigen retained its capacity to bind membrane lipids when it functions as a transcriptional co-activator in the nucleus of meristem cells. Susila et $a l$. found that FT also localizes to TERMINAL FLOWER1 (TFL1)-containing vesicles (12). TFL1 is a homolog of FT that belongs to the PEBP family $(2,7)$, and also binds to phosphatidylglycerol in vitro (3). It acts as a flowering repressor and may be involved in vacuolar trafficking (12), as well as transcriptional regulations (2). It will be interesting to analyze the importance of TFL1 lipid-binding and potential temperature sensitivity in regulating meristem functions antagonistically with FT. Similarly, the potential relevance of temperature-sensitive membrane interaction for members of the PEBP family involved in bud release or tuber formation in other plants will be worth exploring $(2,7)$.

\section{REFERENCES AND NOTES}


1. L. Corbesier et al., science 316, 1030-1033 (2007).

2. Y. Zhu, S. Klasfeld, D. Wagner, Journal of experimental botany 72, 23012311 (2021).

3. H. Susila et al., Science $\mathbf{x x x}, \mathrm{xxx}$ (2021).

4. L. C. Noack, Y. Jaillais, Annual review of plant biology 71, 71-102 (2020).

5. L. A. Colin, Y. Jaillais, Current opinion in plant biology 53, 1-9 (2019).

6. M. Uemura, R. A. Joseph, P. L. Steponkus, Plant physiology 109, 1530 (1995).

7. S. Jin, Z. Nasim, H. Susila, J. H. Ahn, Seminars in cell \& developmental biology 109, 20-30 (2021).

8. Y. Nakamura et al., Nature communications 5, 3553 (2014).

9. D. L. Falcone, J. P. Ogas, C. R. Somerville, BMC plant biology 4, 17 (2004).

10. Q. Li et al., The Plant cell 27, 86-103 (2015).

11. Y. Nakamura et al., iScience 21, 577586 (2019).

12. E. J. Sohn et al., Proceedings of the National Academy of Sciences 104, 18801-18806 (2007).

\section{AUTHORS DECLARE NO COMPETING INTEREST}

FIGURE: Regulation of florigen localization and activity by lipids

Model for the regulation of FLOWERING LOCUS $\mathrm{T}$ (FT, also called florigen) localization and activity by the phospholipids phosphatidylglycerol and phosphatidylcholine. FT expression, localization, movement, and activity are regulated by ambient temperature and the photoperiod, which together influence flowering time.

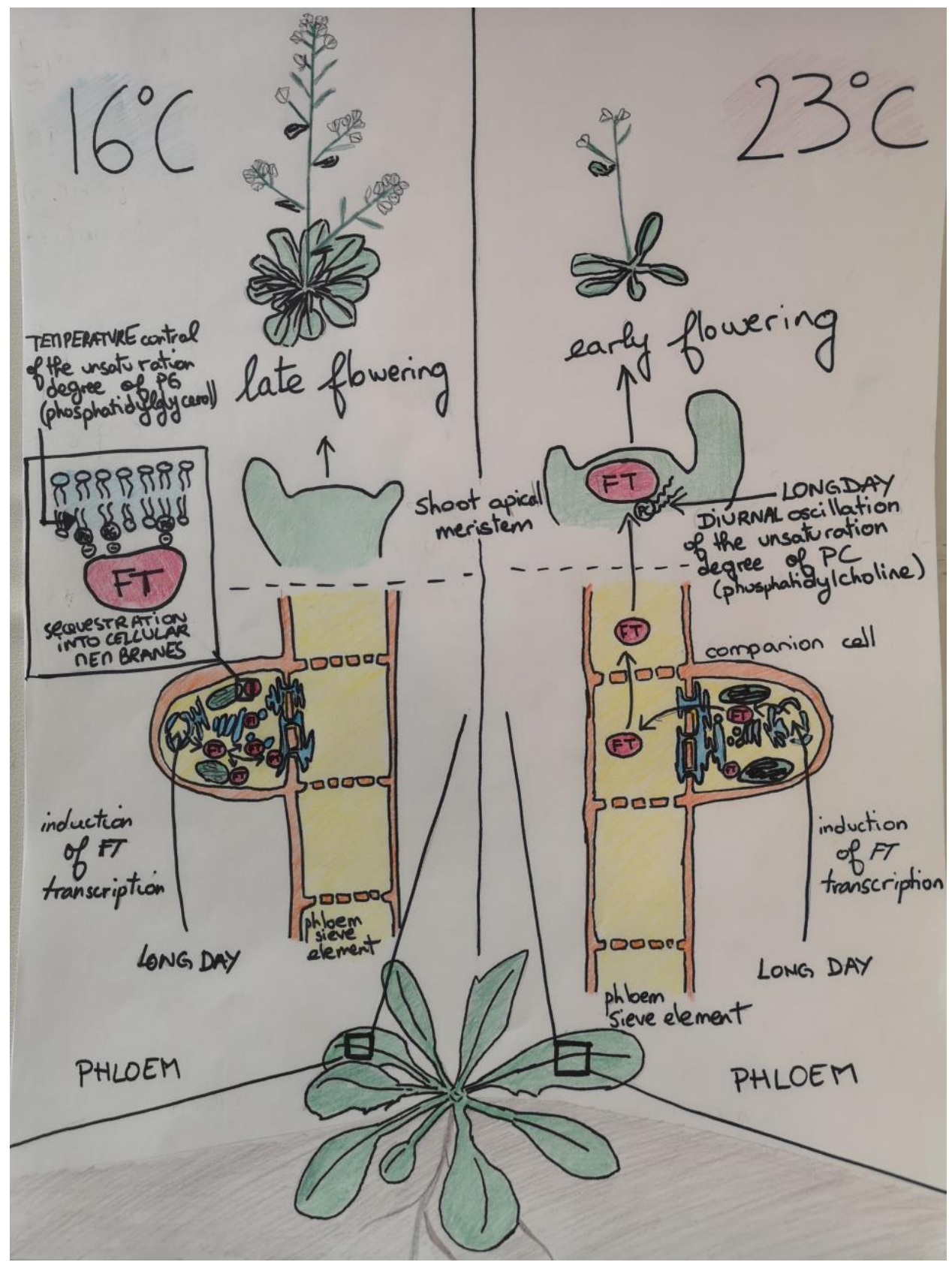

